\title{
PD-L1 and PD-1 expression are correlated with distinctive clinicopathological features in papillary thyroid carcinoma
}

\author{
Yanhua Bai ${ }^{1 * \dagger} \mathbb{D}$, Dongfeng $\mathrm{Niu}^{1 \dagger}$, Xiaozheng Huang ${ }^{1}$, Ling Jia', Qiang Kang ${ }^{1}$, Fangyuan Dou' ${ }^{1}$ Xinqiang Ji ${ }^{2}$, \\ Weicheng Xue', Yiqiang Liu', Zhongwu Li', Qin Feng ${ }^{1}$, Dongmei Lin ${ }^{1}$ and Kennichi Kakudo ${ }^{3}$
}

\begin{abstract}
Background: Immune checkpoint blockade targeting PD-1/PD-L1 has shown efficacy in several types of cancers. However, the correlation between PD-L1/PD-1 expression and the specific clinicopathological features in papillary thyroid carcinoma (PTC) has not been investigated.
\end{abstract}

Methods: We examined the immunohistochemical expression of PD-L1, PD-1, and BRAF V600E on whole-tissue sections from 126 cases of primary PTC more than $1 \mathrm{~cm}$ in size. The correlation between the PD-L1/PD-1 expression and the clinicopathological features was evaluated.

Results: PD-L1 was positively expressed in 53.2\% PTCs, and its expression was positively correlated with rich tumor-infiltrating lymphocytes (TILs), background chronic lymphocytic thyroiditis (CLT), female gender, absence of psammoma bodies, and PD-1 expression. Among these parameters, rich TILs, female gender, and absence of psammoma bodies were independent factors affecting PD-L1 expression on the multivariate logistic regression analysis. PD-1 expression was detected in the TILs and was positively correlated with rich TILs, background CLT, and absence of stromal calcification. Lack of stromal calcification was an independent factor affecting PD-1 expression. Neither PD-L1 nor PD-1 expression showed significant correlation with BRAF V600E expression.

Conclusions: Our results show that the distinctive pathological features of PTCs, including TILs, background CLT, female gender, psammoma bodies, and stromal calcification, are useful parameters for predicting PD-L1 or PD-1 expression.

Keywords: BRAF V600E, Chronic lymphocytic thyroiditis, Papillary thyroid carcinoma, PD-1, PD-L1, Tumor-infiltrating lymphocytes

\section{Background}

PTC is the most common malignancy of the endocrine organs, and its incidence has increased in the past decade. Although PTC overall has a good prognosis, more than $10 \%$ of patients with PTC develop recurrence or distant metastasis after surgery, and some of these patients with succumb to PTC [1]. Although the molecular mechanisms responsible for the initiation and progression of PTC are not fully understood, the mitogen-activated protein kinase (MAPK) pathway has been implicated in the development

\footnotetext{
* Correspondence: byh0425@sina.com

${ }^{\dagger}$ Equal contributors

${ }^{1}$ Key Laboratory of Carcinogenesis and Translational Research (Ministry of Education), Department of Pathology, Peking University Cancer Hospital \& Institute, No. 52 Fucheng Road, Haidian District, Beijing 100142, China Full list of author information is available at the end of the article
}

of more than $70 \%$ of PTCs, as well as BRAF and RAS mutations and gene fusions involving the RET and NTRK1 tyrosine kinase [2-4]. The BRAF mutations play an important role in this process, and the BRAF V600E is the most common alteration. In recent years, a novel mutation-specific antibody targeting BRAF V600E protein has become commercially available. Its specificity and sensitivity to identify the BRAF V600E gene mutation have been verified in many series of tumors, including melanoma and PTC, and it could be used as a surrogate of genetic detection of BRAF V600E mutation [5-7]. This has made it feasible to detect BRAF V600E mutation in PTC using immunohistochemical staining.

As the largest endocrine organ in humans, the thyroid is frequently affected by organ-specific and systemic 
autoimmune diseases, including chronic lymphocytic thyroiditis (CLT), Graves' disease, and Riedel's thyroiditis. Among them, the most frequent condition associated with PTC is chronic lymphocytic thyroiditis $[8,9]$. Many studies have indicated that up to one-third of PTCs arise in the setting of chronic lymphocytic thyroiditis, although these studies tend to lack serologic proof of preexisting thyroiditis [9]. In recent years, immune checkpoint blockade targeting PD-1/PD-L1 has shown efficacy in several types of cancers, and immunohistochemical expression of PD-L1 in cancer has shown significant correlation with clinical outcomes [10-15]. PD-L1, also known as CD274, is a cell-surface glycoprotein of the B7 family. It is expressed on various tissues including solid tumors, and it can facilitate immune evasion and $\mathrm{T}$ cells exhaustion. The interaction of PD-1 on the surface of T cells with PD-L1 on tumor cells suppresses TCR-mediated proliferation and activation, and inhibits $\mathrm{T}$ cell cytolysis. As a result, increased PD-L1 expression by cancer cells is a fundamental host immune escape mechanism [16]. These findings prompted us to examine the relationship between PD-L1/PD-1 expression and a background of CLT in PTC.

The purpose of the present study is to answer the following questions: (1) What is the correlation between the PD-L1/PD-1 expression and the clinicopathological parameters, including a background of CLT in PTCs? (2) What is the relationship between the PD-L1/PD-1 expression and the BRAF V600E expression? (3) What clinicopathological factors affect PD-L1/PD-1 expression in PTCs?

\section{Methods}

\section{Patients and samples}

One hundred twenty-six (126) consecutive patients with a primary PTC measuring more than $10 \mathrm{~mm}$ in diameter who underwent surgical treatment in 2013 at Peking University Cancer Hospital, Beijing, China, were enrolled, including 96 females and 30 males. The patients' age at surgery was $43.94 \pm 13.15$ (mean \pm s.d.). All patients had no evidence of distant metastasis at the time of surgery. All histological subtypes of PTCs were included except microcarcinoma [17].

Sections stained with hematoxylin and eosin from the 126 cases of PTC were histologically evaluated by two pathologists, BY and ND, to confirm the histological subtypes and to examine TILs, psammoma bodies, stromal calcification, bone formation within the tumor mass, and extrathyroid invasion, multifocality as well as background disease. Of these 126 PTCs, 114 cases were conventional type, 8 were follicular variants, 2 were solid variants, 1 was tall cell variant, and 1 case was a Warthin-like tumor variant.

\section{Definition of the Clinicopathological features}

The evaluation of TILs in PTC was according to the evaluation of TILs in breast cancer recommended by an International TILs Working Group in 2014 [18]. Most PTCs have a small number of TILs. In the present study, if TILs occupied $10 \%$ or more of the tumor stroma, it was considered TILs-rich, otherwise it was considered TILs-poor. The definitions of psammoma bodies, stromal calcification, and bone formation were described in detail in our earlier study [19].

The background disease was classified as CLT and non-CLT in the present study. According to Mizukami's definition, CLT was divided into four groups: chronic thyroiditis, oxyphilic; chronic thyroiditis, mixed; chronic thyroiditis, hyperplastic; and chronic thyroiditis, focal [20]. The first group demonstrated classic Hashimoto's disease histology [21]. The second group showed a less lymphoplasmacytic infiltrate than the former, with minimal fibrosis. The third and fourth groups have only a small or focal lymphocytic reaction. Tumor-bearing thyroid tissues demonstrating characteristics of the first and second groups were categorized as CLT in the present study (Fig. 1a and b), and those that did not meet the diagnostic criteria of CLT were categorized as non-CLT, including the third and fourth group in Mizukami's definition, since the latter more likely represented types of non-specific thyroiditis other than autoimmune thyroiditis.

The $\mathrm{pT}, \mathrm{pN}$, and stage groupings were categorized according to the cancer staging of the seventh edition of American Joint Committee on Cancer.

\section{Immunohistochemical analysis}

Formalin-fixed, paraffin-embedded (FFPE) whole-tissue sections were cut to generate 4- $\mu \mathrm{m}$ unstained slides in 126 PTCs, including the tumor center and tumor invasive front, for PD-L1, PD-1, and BRAF V600E staining. All stained sections were examined microscopically and scored independently by three pathologists (BY, ND, and XW).

PD-L1 staining was performed with an anti-human PD-L1 rabbit monoclonal antibody at working solution (SP142; Zhongshan Golden Bridge Biotechnology, Beijing, China) on an automated staining platform (BOND-III, Leica Biosystems Ltd., Newcastle, UK) using a Bond Polymer Refine Detection kit (Leica Biosystems Ltd., Newcastle, UK) according to the manufacturer's instructions. To verify the specificity of the immunostaining, primary antibody was replaced by phosphate-buffered saline from the staining procedure in a set of control sections. Patterns with $5 \%$ or greater positive tumor cells were considered positive, and those with less than 5\% positive cells were considered negative [15].

For PD-1, the FFPE sections were deparaffinized and rehydrated, and then antigen retrieval was carried out in a PTLink machine (Dako, Glostrup, Denmark). Endogenous 


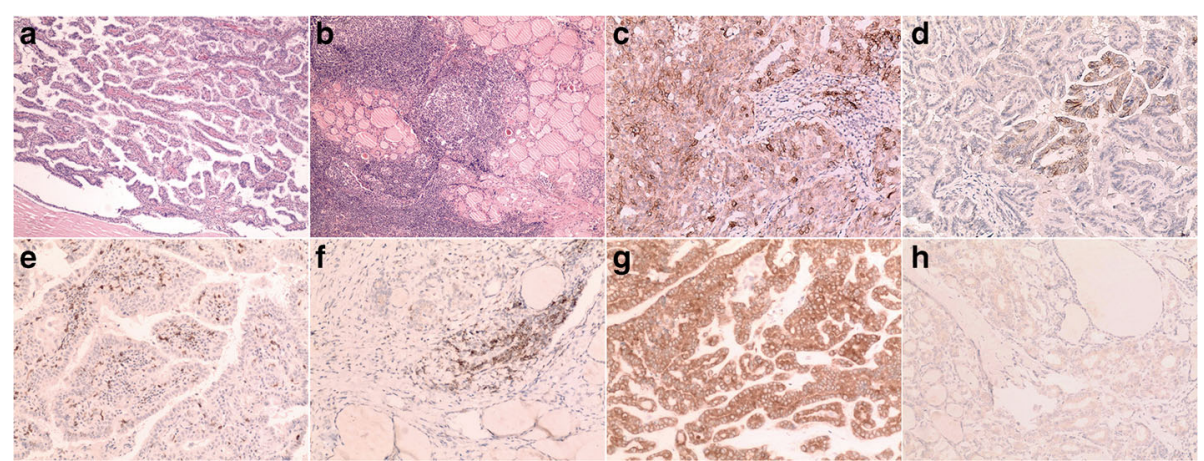

Fig. 1 a, Papillary thyroid carcinoma (PTC) with predominant papillary structures; $\mathbf{b}$, The background thyroid tissue of (a), which showed dense lymphoplasmacytic infiltrates and germinal center formation indicating the presence of chronic lymphocytic thyroiditis; c, PD-L1 was diffusely expressed both on the cell membrane and the cytoplasm. Please note there was also staining on the tumor-infiltrating lymphocytes (TILs), which should not be regarded as positive staining of the tumor cells; $\mathbf{d}$, PD-L1 was focally expressed on the tumor cell membrane; $\mathbf{e}$, PD-1 expression of the TILs was identified in the tumor stroma; $\mathbf{f}$, PD-1 expression was noted in the tumor invasive front; $\mathbf{g}$, BRAF V600E was strongly and diffusely expressed on the tumor cytoplasm; h, Mild but diffuse expression of BRAF V600E was noted on the PTC

peroxidase activity was blocked by incubation in $3 \% \mathrm{H}_{2} \mathrm{O}_{2}$ solution at room temperature for $10 \mathrm{~min}$. Then the sections were incubated with mouse anti-PD-1 monoclonal antibody at working solution (UMAB199; Zhongshan Golden Bridge Biotechnology, Beijing, China) at $37^{\circ} \mathrm{C}$ for $15 \mathrm{~min}$, followed by incubation with anti-mouse IgG-HRP conjugate (EnVision FLEX/HRP; Dako, Glostrup, Denmark) at room temperature for $25 \mathrm{~min}$. Antibody binding was visualized using an EnVision FLEX DAB CHROMOGEN kit (Dako, Glostrup, Denmark) according to the manufacturer's instructions. For general negative controls, the primary antibodies were replaced by phosphate-buffered saline. Positive immunostaining of PD-1 was regarded as staining on $\geq 3$ TILs per high power field (Olympus, BX43) in the hot spot inside the tumor or in the tumor invasive front.

BRAF V600E staining was performed using the anti-BRAF V600E mouse monoclonal primary antibody at working solution (VE1; Ventana Medical Systems, Oro Valley, AZ, USA) on the Benchmark XT platform (Ventana Medical Systems). The specific visualization of V600E-mutated BRAF protein was accomplished using the ultraView Universal DAB Detection Kit (Ventana Medical Systems). Prevalidated BRAF V600E positive and negative PTC specimens were used as positive and negative controls, respectively. According to previous studies together with our experience, the immunoreactivity of V600E-mutated BRAF protein was evaluated as positive if unequivocal diffuse cytoplasmic staining of tumor cells was identified [5, 6, 22]. The staining intensities included weak, moderate, and strong. Focal staining, any type of isolated nuclear staining, and weak staining of single interspersed cells were scored as negative.

\section{Statistical analysis}

Time-independent and categorical data were evaluated using the chi-squared test, continuity correction, or Fisher's exact probability test as appropriate. The multivariate logistic regression model was used to evaluate the multivariate analyses affecting PD-L1 and PD-1 expression. The differences were considered significant when the probability ( $P$-value) was less than 0.05 . Data analysis was performed using SPSS 17.0 statistical software.

\section{Results}

Clinicopathological significance of PD-L1, PD-1, and background of CLT

PD-L1 expression was located in the membrane with or without cytoplasmic staining. The staining pattern was diffuse or focal (Fig. 1c and d). Two of the eight follicular variant PTCs showed focal positive staining of PD-L1. One of the two solid variant PTCs and the only case of Warthin-like tumor demonstrated diffuse staining of PDL1. That case of tall cell variant showed negative PD-L1 staining. Positive PD-L1 expression was identified in 53.2\% PTCs, and positive PD-L1 expression was significantly correlated with rich TILs, female gender, absence of psammoma bodies, and a background of CLT (Table 1).

PD-1 expression was identified in the lymphocytes both within the cancer and in the non-neoplastic thyroid tissue including CLT. Only PD-1 expression on the TILs both inside of and in the invasive front of PTC was counted (Fig. 1e and f). PD-1 was expressed in $84.9 \%$ PTC cases. Expression of PD-1 was significantly correlated with rich TILs, absence of stromal calcification and presence of background CLT (Table 2).

The presence of CLT usually indicates autoimmune disease of the thyroid, although it may overlap with nonspecific lymphocytic thyroiditis. The presence of CLT 
Table 1 The correlation between the PD-L1 expression and other clinicopathological parameters

\begin{tabular}{|c|c|c|c|}
\hline & \multicolumn{2}{|c|}{ PD-L1 expression } & \multirow[t]{2}{*}{$P$-value } \\
\hline & $+(53.2 \%)$ & $-(46.8 \%)$ & \\
\hline \multicolumn{4}{|l|}{ Age } \\
\hline$<45$ years & 33 & 33 & \multirow[t]{2}{*}{0.454} \\
\hline$\geq 45$ years & 34 & 26 & \\
\hline \multicolumn{4}{|l|}{ Gender } \\
\hline Female & 58 & 38 & \multirow[t]{2}{*}{0.004} \\
\hline Male & 9 & 21 & \\
\hline \multicolumn{4}{|l|}{ Tumor size } \\
\hline$\leq 2 \mathrm{~cm}$ & 44 & 39 & \multirow[t]{3}{*}{0.147} \\
\hline$>2$ and $\leq 4 \mathrm{~cm}$ & 19 & 20 & \\
\hline$>4 \mathrm{~cm}$ & 4 & 0 & \\
\hline \multicolumn{4}{|l|}{ Extrathyroid invasion } \\
\hline- & 15 & 15 & \multirow[t]{2}{*}{0.690} \\
\hline+ & 52 & 44 & \\
\hline \multicolumn{4}{|l|}{ pT } \\
\hline $1 b$ & 12 & 13 & \multirow[t]{3}{*}{0.699} \\
\hline 2 & 4 & 2 & \\
\hline 3 & 51 & 44 & \\
\hline \multicolumn{4}{|l|}{ pN } \\
\hline 0 & 25 & 22 & \multirow[t]{3}{*}{0.186} \\
\hline $1 \mathrm{a}$ & 30 & 19 & \\
\hline $1 b$ & 12 & 18 & \\
\hline \multicolumn{4}{|l|}{ Stage grouping } \\
\hline $\mid+\|$ & 8 & 8 & \multirow[t]{3}{*}{0.294} \\
\hline III & 47 & 34 & \\
\hline IVA & 12 & 17 & \\
\hline \multicolumn{4}{|l|}{ Psamomma body } \\
\hline- & 39 & 19 & \multirow[t]{2}{*}{0.003} \\
\hline+ & 28 & 40 & \\
\hline \multicolumn{4}{|l|}{ Stromal calcification } \\
\hline- & 38 & 27 & \multirow[t]{2}{*}{0.220} \\
\hline+ & 29 & 32 & \\
\hline \multicolumn{4}{|l|}{ Bone formation } \\
\hline- & 66 & 57 & \multirow[t]{2}{*}{0.911} \\
\hline+ & 1 & 2 & \\
\hline \multicolumn{4}{|l|}{ Multifocality } \\
\hline Unifocal & 50 & 38 & \multirow[t]{2}{*}{0.212} \\
\hline Multifocal & 17 & 21 & \\
\hline TILs & & & \\
\hline Poor & 39 & 54 & 0.001 \\
\hline Rich & 28 & 5 & \\
\hline Background disease & & & \\
\hline $\mathrm{CLT}$ & 21 & 6 & 0.004 \\
\hline Non-CLT & 46 & 53 & \\
\hline
\end{tabular}

$p T$ pathological tumor category, $p N$ pathological lymph node category, TILs tumor-infiltrating lymphocytes, CLT chronic lymphocytic thyroiditis
Table 2 The correlation between the PD-1 expression and other clinicopathological parameters

\begin{tabular}{llll}
\hline & \multicolumn{2}{l}{ PD-1 expression } & $P$-value \\
\cline { 2 - 3 } & $+(84.9 \%)$ & $-(15.1 \%)$ & \\
\hline Age & 56 & 10 & 0.981 \\
$\quad<45$ years & 51 & 9 & \\
$\quad \geq 45$ years & & & 0.989 \\
Gender & 81 & 15 & \\
$\quad \begin{array}{l}\text { Female } \\
\text { Male }\end{array}$ & 26 & 4 &
\end{tabular}

Tumor size

$\begin{array}{lll}\leq 2 \mathrm{~cm} & 71 & 12 \\ >2 \text { and } \leq 4 \mathrm{~cm} & 34 & 5 \\ >4 \mathrm{~cm} & 2 & 2\end{array}$

0.136

Extrathyroid invasion

$\begin{array}{lll}- & 27 & 3 \\ + & 80 & 16 \\ \text { pT } & & \end{array}$

0.550

$\begin{array}{lll}2 & 6 & 0 \\ 3 & 79 & 16\end{array}$

$\mathrm{pN}$

0

$1 a$

$1 \mathrm{~b}$

41

0.350

Stage grouping

I + ||

16

0.095

III

IVA

69

22

0.477

Psamomma body

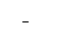

49

0.899

$+$

58

10

Stromal calcification

-

$+$

61

0.004

Bone formation

$-$

105

0.938

$+$

Multifocality

Unifocal

Multifocal

77

0.218

TILS

Poor

Rich

74

0.011

Background disease

$\begin{array}{lll}\text { CLT } & 27 & 0 \\ \text { Non-CLT } & 80 & 19\end{array}$

0.030

$p T$ pathological tumor category, $p N$ pathological lymph node category, TILs tumor-infiltrating lymphocytes, CLT chronic lymphocytic thyroiditis 
was significantly correlated with both PD-L1 and PD-1 expression. PD-L1 expression was in 77.8 and $46.5 \%$ cases of PTCs with and without CLT in the background thyroid tissue, respectively $(P=0.004$, Table 1$)$. PD-1 expression was in 100 and $80.8 \%$ PTCs with and without CLT in the background thyroid tissue, respectively $(P=0.030$, Table 2 ). The presence of CLT was also correlated with negative extrathyroid invasion, low stage grouping, and rich TILs $(P=0.020,0.032$, and 0.001 , respectively, Table 3$)$.

\section{Correlation between PD-L1, PD-1, and BRAF V600E expression}

Positive BRAF V600E staining (Fig. $1 \mathrm{~g}$ and $\mathrm{h}$ ) was seen in $76.2 \%$ of 126 cases of PTCs. BRAF V600E staining was not correlated with PD-L1 or PD-1 expression (Fig. 2a and b). PD-L1 and PD-1 expression were significantly correlated with each other $(P=0.011$, Fig. $2 \mathrm{c})$. There was no significant correlation between BRAF V600E expression and TILs (Fig. 2d).

\section{Clinicopathological features affecting PD-L1 and PD-1 expression}

Clinicopathological features that are correlated with PD-L1 expression including rich TILs, a background disease of CLT, female gender, and the absence of psammoma bodies, together with PD-1 expression, were subsequently analyzed using the multivariate logistic regression analysis model. Among them, rich TILs, female gender, and the absence of psammoma bodies, were independent factors to predict PD-L1 expression (Table 4). Rich TILs, a background disease of CLT, absence of stromal calcification, together with PD-L1 expression were analyzed using the multivariate logistic regression analysis model, to detect independent factors predicting PD-1 expression. Among them, only the absence of stromal calcification was independent predicting factor for PD-1 expression (Table 5).

\section{Discussion}

In this study, we examined the expression of PD-L1 and PD-1 in 126 cases of PTCs and analyzed their association with clinicopathological parameters. Both PD-L1 and PD-1 expression were correlated with CLT in the background thyroid tissue. Many studies have indicated that up to onethird of PTCs arose in a background of chronic thyroiditis. However, these studies did not have serologic evidence of preexisting thyroiditis [9]. In our cases, $21.4 \%$ of PTCs occurred in the thyroid with a background of CLT, which was less frequent than previously reported. The discrepancy between ours and those previously reported was most likely due to the fact that small or focal lymphocytes infiltration was not regarded as CLT in our study, since they most likely represent non-specific thyroiditis other than autoimmune thyroiditis. Because PTC and thyroiditis are both common conditions, the coexistence of them is more likely
Table 3 The correlation between the background disease and other clinicopathological parameters

\begin{tabular}{|c|c|c|c|}
\hline & \multicolumn{2}{|c|}{ Background disease } & \multirow[t]{2}{*}{$P$-value } \\
\hline & $\overline{\mathrm{CLT}}$ & Non-CLT & \\
\hline \multicolumn{4}{|l|}{ Age } \\
\hline$<45$ years & 14 & 52 & \multirow[t]{2}{*}{0.950} \\
\hline$\geq 45$ years & 13 & 47 & \\
\hline \multicolumn{4}{|l|}{ Gender } \\
\hline Female & 24 & 72 & \multirow[t]{2}{*}{0.081} \\
\hline Male & 3 & 27 & \\
\hline \multicolumn{4}{|l|}{ Tumor size } \\
\hline$\leq 2 \mathrm{~cm}$ & 18 & 65 & \multirow[t]{3}{*}{0.561} \\
\hline$>2$ and $\leq 4 \mathrm{~cm}$ & 9 & 30 & \\
\hline$>4 \mathrm{~cm}$ & 0 & 4 & \\
\hline \multicolumn{4}{|l|}{ Extrathyroid invasion } \\
\hline- & 11 & 19 & \multirow[t]{2}{*}{0.020} \\
\hline+ & 16 & 80 & \\
\hline \multicolumn{4}{|l|}{ pT } \\
\hline $1 b$ & 9 & 16 & \multirow[t]{3}{*}{0.089} \\
\hline 2 & 2 & 4 & \\
\hline 3 & 16 & 79 & \\
\hline \multicolumn{4}{|l|}{$\mathrm{pN}$} \\
\hline 0 & 11 & 36 & \multirow[t]{3}{*}{0.203} \\
\hline $1 a$ & 13 & 36 & \\
\hline $1 b$ & 3 & 27 & \\
\hline \multicolumn{4}{|l|}{ Stage grouping } \\
\hline $1+\|$ & 7 & 9 & \multirow[t]{3}{*}{0.032} \\
\hline III & 17 & 64 & \\
\hline IVA & 3 & 26 & \\
\hline \multicolumn{4}{|l|}{ Psamomma body } \\
\hline- & 10 & 48 & \multirow[t]{2}{*}{0.290} \\
\hline+ & 17 & 51 & \\
\hline \multicolumn{4}{|l|}{ Stromal calcification } \\
\hline- & 16 & 49 & \multirow[t]{2}{*}{0.368} \\
\hline+ & 11 & 50 & \\
\hline \multicolumn{4}{|l|}{ Bone formation } \\
\hline- & 27 & 96 & \multirow[t]{2}{*}{0.839} \\
\hline+ & 0 & 3 & \\
\hline \multicolumn{4}{|l|}{ Multifocality } \\
\hline Unifocal & 19 & 69 & \multirow[t]{2}{*}{0.946} \\
\hline Multifocal & 8 & 30 & \\
\hline \multicolumn{4}{|l|}{ TILs } \\
\hline Poor & 5 & 88 & \multirow[t]{2}{*}{0.001} \\
\hline Rich & 22 & 11 & \\
\hline
\end{tabular}

$p T$ pathological tumor category, $p N$ pathological lymph node category, TILs tumor-infiltrating lymphocytes, CLT chronic lymphocytic thyroiditis 

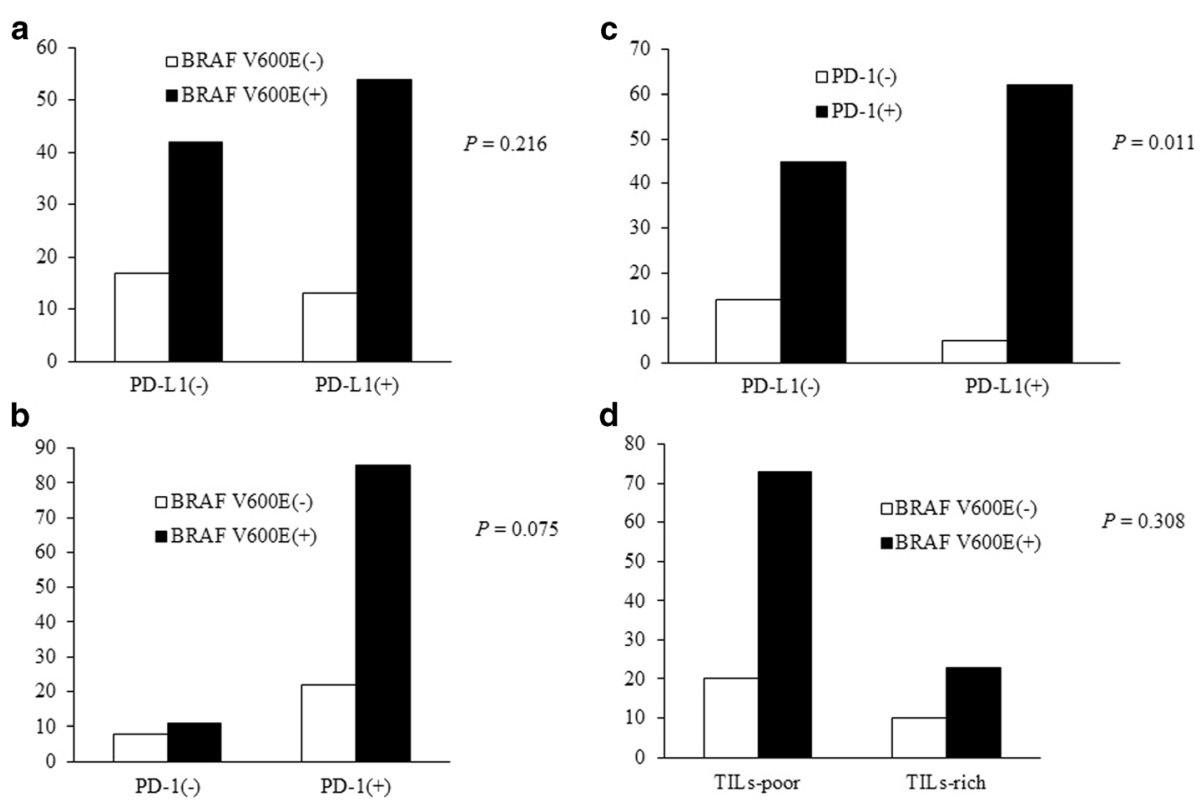

Fig. 2 a, The correlation between BRAF V600E and PD-L1 expression; b, The correlation between BRAF V600E and PD-1 expression; c, The correlation between PD-L1 and PD-1 expression; $\mathbf{d}$, the correlation between BRAF V600E expression and tumor-infiltrating lymphocytes (TILs)

to be a coincidental incident than an etiologic relationship $[8,9]$. For patients with CLT, T lymphocytes were of predominantly suppressor type within the thyroid, whereas there were mostly helper $\mathrm{T}$ cells in the periphery blood $[23$, 24]. A previous study has indicated that positive thyroglobulin antibody in the serum of PTC patients was associated with lower tumor stage on univariate analysis and associated with a favorable clinical outcome $[25,26]$, which

Table 4 Multivariate logistic analysis of examined clinicopathological parameters influencing PD-L1 expression

\begin{tabular}{|c|c|c|c|}
\hline Parameter & OR & $95 \% \mathrm{Cl}$ & $P$-value \\
\hline \multicolumn{4}{|l|}{ Gender } \\
\hline Male & 1.000 & / & 0.019 \\
\hline Female & 3.393 & $1.225-9.395$ & \\
\hline \multicolumn{4}{|c|}{ Background disease } \\
\hline Non-CLT & 1.000 & / & 0.803 \\
\hline CLT & 1.206 & $0.276-5.265$ & \\
\hline \multicolumn{4}{|c|}{ Psammoma body } \\
\hline- & 1.000 & / & 0.002 \\
\hline+ & 0.263 & $0.112-0.614$ & \\
\hline \multicolumn{4}{|c|}{ PD-1 expression } \\
\hline- & 1.000 & / & 0.067 \\
\hline+ & 3.099 & $0.923-10.400$ & \\
\hline \multicolumn{4}{|l|}{ TILs } \\
\hline Poor & 1.000 & / & 0.028 \\
\hline Rich & 5.036 & $1.189-21.340$ & \\
\hline
\end{tabular}

was similar to our present finding (PTC with a background of CLT correlated with lower tumor stage and negative extra-thyroidal invasion). Both PD-L1 and PD-1 were highly expressed in PTC cases harboring a background CLT in our study, although the presence of a CLT background was not an independent factor affecting PD-L1 or PD-1 expression. It should be noticed that the presence of CLT may possibly influence the efficacy of immunotherapy using antibodies against PD-L1/PD-1, because of its intimate correlation with both PD-1 and PD-L1 expression. Further studies are required to answer this question.

Table 5 Multivariate logistic analysis of examined clinicopathological parameters influencing PD-1 expression

\begin{tabular}{|c|c|c|c|}
\hline Parameter & OR & $95 \% \mathrm{Cl}$ & $P$-value \\
\hline \multicolumn{4}{|c|}{ Background disease } \\
\hline Non-CLT & 1.000 & / & 0.998 \\
\hline CLT & 9.155 E7 & / & \\
\hline \multicolumn{4}{|c|}{ Stromal calcification } \\
\hline- & 1.000 & / & 0.020 \\
\hline+ & 0.237 & $0.070-0.799$ & \\
\hline \multicolumn{4}{|c|}{ PD-L1 expression } \\
\hline- & 1.000 & / & 0.115 \\
\hline+ & 2.542 & $0.796-8.120$ & \\
\hline \multicolumn{4}{|l|}{ TILs } \\
\hline Poor & 1.000 & / & 0.998 \\
\hline Rich & 4.455E7 & / & \\
\hline
\end{tabular}


Expression of PD-L1 and that of PD-1 were significantly correlated each other in the present study, and PD-1 expression may influence the clinical significance of PD-L1 expression, and vice versa. A previous study has classified the tumor microenvironments into four groups based on PD-L1 expression and TILs [27]. Different immunotherapy strategies should be applied in different groups [27]. The expression status of PD-1 on the TILs is a more direct indicator than TILs itself to reflect the tumor immune suppression status, and thus clarifying the PD-L1 and PD-1 expression status of a tumor could help to select proper tumor immunotherapy approaches.

Recently, high expression of PD-L1 was reported by Chowdhury et al. to be correlated with invasiveness of PTC and poor disease-free survival [28]. Our cases were all PTCs with tumor size $>1 \mathrm{~cm}$, and of our 126 cases, 124 showed an invasive growth pattern. Extrathyroid invasion is a useful parameter to predict tumor recurrence or tumor death [1]. However, the correlation between expression of PD-L1 and extrathyroid invasion did not reach a statistical significance both in Chowdhury's research and in our study [28]. Although we did not examine the direct correlation between PD-L1 expression and disease-free survival, the clinical correlation of PD-L1 expression did not suggest a possible poor clinical outcome in PD-L1 positive cases. Expression of PD-L1 was correlated with the female gender and the absence of psammoma bodies, and expression of PD-1 was correlated with the absence of stromal calcification. Psammoma bodies were considered as being formed by calcification subsequent to the necrosis of tumor thrombi [29]. Carcangiu et al. reported that PTC patients with psammoma bodies were affected more often by persistent disease and had a higher incidence of lymph node and pulmonary metastasis [30]. Our previous study indicated that PTC patients with psammoma bodies, compared to those without, had poorer disease-free survival [19]. The significant correlation between PD-L1 expression and absence of psammoma bodies may indicate that PD-L1 expression is possibly associated with a favorable clinical outcome. The stromal calcification was regarded as being formed due to the deposition of calcium phosphate in the fibrous stroma. The formation of both psammoma bodies and stromal calcification requires some time and may indicate a relatively long clinical course. The positive correlation between PD-L1 expression and the absence of psammoma bodies, and between PD-1 expression and the absence of stromal calcification, may indicate that both PD-L1 and PD-1 expressions are correlated with a short survival clinical course, and they are probably involved in the tumor growth, as indicated in ovarian cancer [31]. Ahn recently used tissue microarrays to investigate 407 primary thyroid cancers for PD-L1 expression, and no significant association was found between PD-L1 expression and clinicopathologic variables, disease progression, and oncogenic mutation [32], which was different from our finding. Further studies using whole-tissue sections are required to confirm or disprove the clinical correlations of PD-L1 and PD-1.

The constitutively active BRAF V600E protein has been associated with worse clinical outcomes in thyroid cancer [33]. Angell et al. reported that BRAF V600E was associated with increased PD-L1 expression in PTC [34]. In our study, the correlation between PD-L1 and BRAF V600E did not reach a statistical significance. This could be explained partly by the sample selection. In Angell et al.'s 33 cases of PTC, $78.8 \%$ were pT1 or pT2 cases and their cases in Stage I totaled 60.7\%. However, our 126 cases of PTC were consecutive cases, with $19.8 \%$ of pT1 and $12.7 \%$ of Stage I and II. Another parameter affecting the correlation between PD-L1 and BRAF V600E was the presence of a background CLT. After excluding PTC cases with a background of CLT, BRAF V600E was positively correlated with PD-L1 expression (manuscript in progress).

\section{Conclusion}

We examined the expression of PD-L1, PD-1, and BRAF V600E in 126 cases of PTC, and found that distinctive pathological features in PTC, including TILs, a background of CLT, psammoma bodies, and stromal calcification, were useful parameters for predicting PD-L1 or PD-1 expression, which was a novel finding and should be highlighted in the forthcoming era of immunotherapy. Further studies are necessary to determine the prognostic and therapeutic value of these findings in PTCs.

\section{Abbreviations \\ Cl: Confidence interval; CLT: Chronic lymphocytic thyroiditis; FFPE: Formalin-fixed, paraffin-embedded; OR: Odds ratio; pN: Pathological lymph node category; pT: Pathological tumor category; PTC: Papillary thyroid carcinoma; TILs: Tumor-infiltrating lymphocytes}

\section{Acknowledgements \\ None.}

\section{Funding}

This work was supported by the National Nature Science Foundation of China (No. 81202114 and No. 81301879), and the Scientific Research Foundation for Returned Scholars, Ministry of Education of China, for Yanhua Bai.

\section{Availability of data and materials}

The raw data are available upon request on the following e-mail address: byh0425@sina.com.

\section{Authors' contributions}

$B Y, L Z, L D$, and KK conceived and designed the experiments. DF reviewed the patients' oncological data and selected the cases. $H X, J L$, and $K Q$ performed the experiments. BY, ND, and XW reviewed the slides; JX, LY, and FQ analyzed the data. BY and ND wrote the manuscript. All authors were involved in data interpretation, writing the paper, and had final approval of the submitted and published manuscript. 


\section{Ethics approval and consent to participate}

This study was approved by the ethics committee of Peking University Cancer Hospital \& Institute (Permission number 2013KT34). Additional patient consent for this retrospective study was not required.

\section{Consent for publication}

Not applicable.

\section{Competing interests}

The authors declare that they have no competing interests.

\section{Publisher's Note}

Springer Nature remains neutral with regard to jurisdictional claims in published maps and institutional affiliations.

\section{Author details}

'Key Laboratory of Carcinogenesis and Translational Research (Ministry of Education), Department of Pathology, Peking University Cancer Hospital \& Institute, No. 52 Fucheng Road, Haidian District, Beijing 100142, China. ${ }^{2}$ Key Laboratory of Carcinogenesis and Translational Research (Ministry of Education), Department of Medical Statistics, Peking University Cancer Hospital \& Institute, No. 52 Fucheng Road, Haidian District, Beijing 100142, China. ${ }^{3}$ Department of Pathology, Nara Hospital, Kindai University Faculty of Medicine, Ikoma-city, Nara 630-0293, Japan.

Received: 28 March 2017 Accepted: 25 September 2017 Published online: 03 October 2017

\section{References}

1. Bai Y, Kakudo K, Li Y, et al. Subclassification of non-solid-type papillary thyroid carcinoma identification of high-risk group in common type. Cancer Sci. 2008;99:1908-15

2. Cancer Genome Atlas Research N. Integrated genomic characterization of papillary thyroid carcinoma. Cell. 2014;159:676-90.

3. Kimura ET, Nikiforova MN, Zhu Z, Knauf JA, Nikiforov YE, Fagin JA. High prevalence of BRAF mutations in thyroid cancer: genetic evidence for constitutive activation of the RET/PTC-RAS-BRAF signaling pathway in papillary thyroid carcinoma. Cancer Res. 2003;63:1454-7.

4. Xing MBRAF. mutation in thyroid cancer. Endocr Relat Cancer. 2005;12:245-62.

5. Liu H, Li Z, Wang Y, et al. Immunohistochemical detection of the BRAF V600E mutation in melanoma patients with monoclonal antibody VE1. Pathol Int. 2014:64:601-6.

6. Sun J, Zhang J, Lu J, et al. Immunohistochemistry is highly sensitive and specific for detecting the BRAF V600E mutation in papillary thyroid carcinoma. Int J Clin Exp Pathol. 2015;8:15072-8.

7. Pyo JS, Sohn JH, Kang GBRAF. Immunohistochemistry Using Clone VE1 is Strongly Concordant with BRAF(V600E) Mutation Test in Papillary Thyroid Carcinoma. Endocr Pathol. 2015;26:211-7.

8. Ozaki O, Ito K, Kobayashi K, Toshima K, Iwasaki H, Yashiro T. Thyroid carcinoma in Graves' disease. World J Surg. 1990;14:437-40. discussion 40-1

9. Tamimi DM. The association between chronic lymphocytic thyroiditis and thyroid tumors. Int J Surg Pathol. 2002;10:141-6.

10. Jia M, Feng W, Kang $S$, et al. Evaluation of the efficacy and safety of anti-PD1 and anti-PD-L1 antibody in the treatment of non-small cell lung cancer (NSCLC): a meta-analysis. J Thorac Dis. 2015;7:455-61.

11. Massari F, Santoni M, Ciccarese C, et al. PD-1 blockade therapy in renal cell carcinoma: current studies and future promises. Cancer Treat Rev. 2015;41:114-21.

12. Zhang T, Xie J, Arai S, et al. The efficacy and safety of anti-PD-1/PD-L1 antibodies for treatment of advanced or refractory cancers: a meta-analysis. Oncotarget. 2016;7:73068-79.

13. Ilie M, Falk AT, Butori $C$, et al. PD-L1 expression in basaloid squamous cell lung carcinoma: Relationship to PD-1+ and CD8+ tumor-infiltrating T cells and outcome. Mod Pathol. 2016:29:1552-64.

14. Leite KR, Reis ST, Junior JP, et al. PD-L1 expression in renal cell carcinoma clear cell type is related to unfavorable prognosis. Diagn Pathol. 2015:10:189.

15. Li Z, Lai $Y$, Sun L, et al. PD-L1 expression is associated with massive lymphocyte infiltration and histology in gastric cancer. Hum Pathol. 2016;55:182-9.
16. Iwai Y, Ishida M, Tanaka Y, Okazaki T, Honjo T, Minato N. Involvement of PD-L1 on tumor cells in the escape from host immune system and tumor immunotherapy by PD-L1 blockade. Proc Natl Acad Sci U S A. 2002;99:12293-7.

17. Zuo $H$, Tang $W$, Yasuoka $H$, et al. A review of 227 cases of small papillary thyroid carcinoma. Eur J Surg Oncol. 2007;33:370-5.

18. Salgado R, Denkert C, Demaria $\mathrm{S}$, et al. The evaluation of tumor-infiltrating lymphocytes (TILS) in breast cancer: recommendations by an International TILs Working Group 2014. Ann Oncol. 2015;26:259-71.

19. Bai Y, Zhou G, Nakamura M, et al. Survival impact of psammoma body, stromal calcification, and bone formation in papillary thyroid carcinoma. Mod Pathol. 2009:22:887-94.

20. Mizukami $Y$, Michigishi T, Kawato M, et al. Chronic thyroiditis: thyroid function and histologic correlations in 601 cases. Hum Pathol. 1992:23:980-8.

21. Li Y, Zhou G, Ozaki T, et al. Distinct histopathological features of Hashimoto's thyroiditis with respect to lgG4-related disease. Mod Pathol. 2012;25:1086-97.

22. Koperek O, Kornauth C, Capper D, et al. Immunohistochemical detection of the BRAF V600E-mutated protein in papillary thyroid carcinoma. Am J Surg Pathol. 2012;36:844-50

23. Iwatani $Y$, Amino N, Mori $\mathrm{H}$, et al. T lymphocyte subsets in autoimmunethyroid diseases and subacute thyroiditis detected with monoclonal antibodies. J Clin Endocrinol Metab. 1983:56:251-4.

24. Mclntosh RS, Watson PF, Weetman AP. Analysis of the T cell receptor $V$ alpha repertoire in Hashimoto's thyroiditis: evidence for the restricted accumulation of CD8+ T cells in the absence of CD4+ T cell restriction. J Clin Endocrinol Metab. 1997:82:1140-6.

25. McLeod DS, Cooper DS, Ladenson PW, et al. Prognosis of differentiated thyroid cancer in relation to serum thyrotropin and thyroglobulin antibody status at time of diagnosis. Thyroid. 2014;24:35-42.

26. Spencer CA. Clinical review: Clinical utility of thyroglobulin antibody (TgAb) measurements for patients with differentiated thyroid cancers (DTC). J Clin Endocrinol Metab. 2011;96:3615-27.

27. Teng MW, Ngiow SF, Ribas A, Smyth MJ. Classifying Cancers Based on T-cell Infiltration and PD-L1. Cancer Res. 2015;75:2139-45.

28. Chowdhury S, Veyhl J, Jessa F, et al. Programmed death-ligand 1 overexpression is a prognostic marker for aggressive papillary thyroid cancer and its variants. Oncotarget. 2016;7:32318-28.

29. Johannessen JV, Sobrinho-Simoes M. The origin and significance of thyroid psammoma bodies. Lab Investig. 1980:43:287-96.

30. Carcangiu ML, Zampi G, Pupi A, Castagnoli A, Rosai J. Papillary carcinoma of the thyroid. A clinicopathologic study of 241 cases treated at the University of Florence, Italy. Cancer. 1985;55:805-28.

31. Clark CA, Gupta HB, Sareddy G, et al. Tumor-Intrinsic PD-L1 Signals Regulate Cell Growth, Pathogenesis, and Autophagy in Ovarian Cancer and Melanoma. Cancer Res. 2016;76:6964-74.

32. Ahn $\mathrm{S}, \mathrm{Kim} \mathrm{TH}, \mathrm{Kim} \mathrm{SW}$, et al Comprehensive screening for PD-L1 expression in thyroid cancer. Endocr Relat Cancer. 2017;24:97-106.

33. Xing M, Alzahrani AS, Carson KA, et al. Association between BRAF V600E mutation and recurrence of papillary thyroid cancer. J Clin Oncol. 2015:33:42-50.

34. Angell TE, Lechner MG, Jang JK, Correa AJ, LoPresti JS, Epstein ALBRAF. V600E in papillary thyroid carcinoma is associated with increased programmed death ligand 1 expression and suppressive immune cell infiltration. Thyroid. 2014;24:1385-93.

\section{Submit your next manuscript to BioMed Central and we will help you at every step:}

- We accept pre-submission inquiries

- Our selector tool helps you to find the most relevant journal

- We provide round the clock customer support

- Convenient online submission

- Thorough peer review

- Inclusion in PubMed and all major indexing services

- Maximum visibility for your research

Submit your manuscript at www.biomedcentral.com/submit
Biomed Central 